\title{
Alcohol Induces Synaptotagmin 1 Expression in Neurons via Activation of Heat Shock Factor 1
}

\author{
Florence P. Varodayan ${ }^{a, b}$, Leonardo Pignatarob ${ }^{b}$, and Neil L. Harrison ${ }^{b, c}$ \\ Florence P. Varodayan: fpv2102@columbia.edu; Leonardo Pignataro: Ip2391@columbia.edu; Neil L. Harrison: \\ nh2298@columbia.edu

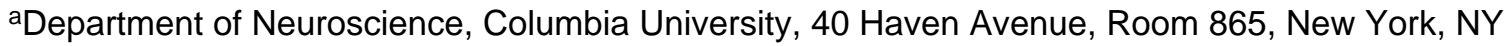 \\ 10032, USA \\ ${ }^{b}$ Department of Anesthesiology, Columbia University, 622 West 168th St, PH-511, New York, NY \\ 10032, USA \\ 'Department of Pharmacology, Columbia University, 630 West $168^{\text {th }}$ Street, PH 7W 318, New \\ York, NY 10032, USA
}

\section{Abstract}

\begin{abstract}
Many synapses within the central nervous system are sensitive to ethanol. Although alcohol is known to affect the probability of neurotransmitter release in specific brain regions, the effects of alcohol on the underlying synaptic vesicle fusion machinery have been little studied. To identify a potential pathway by which ethanol can regulate neurotransmitter release, we investigated the effects of acute alcohol exposure (1-24 hours) on the expression of the gene encoding Synaptotagmin 1 (Syt1), a synaptic protein that binds calcium to directly trigger vesicle fusion. Sytl was identified in a microarray screen as a gene that may be sensitive to alcohol and heat shock. We found that Syt $1 \mathrm{mRNA}$ and protein expression are rapidly and robustly up-regulated by ethanol in mouse cortical neurons, and that the distribution of Syt1 protein along neuronal processes is also altered. Sytl mRNA up-regulation is dependent on the activation of the transcription factor heat shock factor 1 (HSF1). The transfection of a constitutively active $H s f l$ construct into neurons stimulates Sytl transcription, while transfection of $H s f l$ siRNA or a constitutively inactive $H s f l$ construct into neurons attenuates the induction of Sytl by ethanol. This suggests that the activation of HSF1 can induce Syt 1 expression and that this may be a mechanism by which alcohol regulates neurotransmitter release during brief exposures. Further analysis revealed that a subset of the genes encoding the core synaptic vesicle fusion (SNARE) proteins share this property of induction by ethanol, suggesting that alcohol may trigger a specific coordinated adaptation in synaptic function. This molecular mechanism could explain some of the changes in synaptic function that occur following alcohol administration, and may be an important step in the process of neuronal adaptation to alcohol.
\end{abstract}

\footnotetext{
(c) 2011 IBRO. Published by Elsevier Ltd. All rights reserved.

Corresponding author: Neil L. Harrison, Department of Anesthesiology, 622 West 168th St, PH-511, New York, NY 10032, USA., Tel.: 1-212-304-6459., Fax: 1-212-305-3675., nh2298@ columbia.edu.

Publisher's Disclaimer: This is a PDF file of an unedited manuscript that has been accepted for publication. As a service to our customers we are providing this early version of the manuscript. The manuscript will undergo copyediting, typesetting, and review of the resulting proof before it is published in its final citable form. Please note that during the production process errors may be discovered which could affect the content, and all legal disclaimers that apply to the journal pertain.
} 


\section{Keywords}

alcohol; synaptotagmin 1 (Syt1); heat shock factor 1 (HSF1); SNARE proteins; gene expression; cortical neurons

Synapses are generally regarded as the most sensitive sites of ethanol action within the central nervous system. While the majority of research has focused on the post-synaptic effects of alcohol on a variety of neurotransmitter receptors (Lovinger, 1997; Harris, 1999), a growing body of evidence suggests that acute and chronic ethanol treatment can also directly modulate neurotransmitter release in a variety of different brain regions (Siggins et al., 2005; Roberto et al., 2006; Weiner and Valenzuela, 2006).

Electrophysiological work by several groups indicates that acute application of ethanol increases pre-synaptic $\gamma$-aminobutyric acid (GABA) release in the CA1 region of the hippocampus (Carta et al., 2003), nucleus accumbens (Crowder and Weiner, 2002), cerebellum (Carta et al., 2004) and the central amygdala (Roberto et al., 2003), as revealed by increases in the frequency of spontaneous and miniature inhibitory post-synaptic currents (IPSCs). Similar studies suggest that ethanol decreases glutamate release in spinal motoneurons (Ziskind-Conhaim et al., 2003). In addition, investigators have used confocal microscopy in hippocampal slices pre-loaded with the lipophilic dye FM1-43 to reveal the inhibition of glutamate release by ethanol (Maldve et al., 2004). In light of these and many other studies, it is surprising that there has been little work directed specifically towards investigating how alcohol may regulate the expression of genes that encode the components of the synaptic terminal and proteins that control vesicle fusion.

There are a growing number of alcohol-responsive genes (ARGs), most of which have been identified using microarray screening and then confirmed using other approaches (Lewohl et al., 2000; Mulligan et al., 2006). One such candidate ARG recently identified in a microarray screen is the synaptic vesicle membrane protein synaptotagmin 1 (Syt1; Pignataro et al., 2007). Syt1 acts as a calcium sensor within the space immediately adjacent to the site of synaptic vesicle fusion (Brose et al., 1992), and therefore functions as a critical intermediary in the process of action potential-dependent neurotransmitter release. Syt1 is expressed widely across the forebrain, midbrain and in most brainstem and spinal cord neurons (Xu et al., 2007). In the presence of calcium, Syt1 binds to both v-SNAREs (VAMP, also known as synaptobrevin) and plasma membrane phospholipids (Martens et al., 2007). This brings the two membranes together to promote zippering of VAMP and target tSNAREs (SNAP-25, syntaxin-1a) on the plasma membrane, and thus triggers vesicle fusion leading to neurotransmitter release. Synaptophysin 1 regulates this process by associating with VAMP to prevent premature formation of the core SNARE fusion complex (Valtorta et al., 2004).

As Syt1 is intimately involved in the ultimate step of synaptic vesicle fusion, it seems obvious that changes in its expression levels have the potential to alter neurotransmitter release. Syt1-deficient mice show impairment in the fast synchronous component of evoked excitatory post-synaptic currents (EPSCs) in hippocampal neurons (Geppert et al., 1994) and these mice have attenuated IPSCs in cortical neurons (Xu et al., 2007). The over-expression of Syt 1 in mouse hippocampal cultures increases the probability of evoked vesicle release (Han et al., 2004). Since Syt1 is a key regulator of synaptic vesicle fusion, we reasoned that a careful study of the effects of alcohol on the regulation of Syt1 expression might reveal 
additional molecular mechanisms by which alcohol can affect neurotransmitter release dynamics and synaptic transmission.

\section{Experimental Procedures}

\subsection{Cell culture and Immunocytochemistry}

Cortical neurons were cultured from embryonic day 17-18 C57BL/6 mice (Charles River Laboratories, Wilmington, MA) as previously described (Huettner and Baughman, 1986) with modifications (Ma et al., 2004). Lower density cortex cultures were also established and maintained using techniques similar to those used for hippocampal neurons (Banker and Goslin, 1991). The lower density cultures were used for immunocytochemistry experiments 7-11 days in vitro (DIV). Immunostaining was performed with a rabbit polyclonal anti-Syt1 antibody (1:200, Synaptic Systems, Goettingen, Germany) and a mouse monoclonal anti- $\alpha-$ tubulin antibody (1:10000, clone DM1A, Sigma-Aldrich, St. Louis, MO). Cells were mounted with ProLong Gold anti-fade reagent containing the nuclear stain DAPI (Molecular Probes, Eugene, OR). Images were acquired with an inverted Zeiss Axiovert 200 confocal microscope (LSM 510 META; Carl Zeiss Meditech, Thornwood, NY) equipped with diode $(405 \mathrm{~nm})$, argon $(458,477,488,514 \mathrm{~nm}), \mathrm{HeNe} 1(543 \mathrm{~nm})$ and $\mathrm{HeNe} 2(633 \mathrm{~nm})$ lasers.

\subsection{Ethanol and Heat Stress treatments}

Cortical neurons (7-11 DIV) were exposed to ethanol (10-150 mM; Sigma-Aldrich) for specific time periods $(15 \mathrm{~m}-24 \mathrm{~h})$, by addition directly to the culture medium. Cells were subjected to heat stress by transferring them to an incubator set at $42^{\circ} \mathrm{C}$ for a period of $1-2 \mathrm{~h}$.

\subsection{Quantitative Real-time Polymerase Chain Reaction (qPCR) analyses of mRNA Levels}

Total RNA was isolated from cultured neurons using TRIzol (Invitrogen, Carlsbad, CA). cDNA was prepared from total RNA with the iScript cDNA synthesis kit (Bio-Rad, Hercules, CA). For cDNA preparation, reactions were performed in a final volume of $20 \mu \mathrm{l}$; primers were annealed at $25^{\circ} \mathrm{C}$ for $5 \mathrm{~min}$, RNA was reverse transcribed at $42^{\circ} \mathrm{C}$ for $90 \mathrm{~min}$, followed by heat-inactivation at $95^{\circ} \mathrm{C}$ for 5 minutes and the reaction mixtures were stored at $-20^{\circ} \mathrm{C}$. The first-strand reverse transcribed cDNA was then used as a template for PCR amplification using the appropriate specific primer pairs listed below. Quantitative "real time" polymerase chain reaction (qPCR) was carried out with iQ SYBR Green Supermix (Bio-Rad) as previously described (Ma et al., 2004). In preliminary experiments, Syt1 cDNA concentration was normalized against Actb and Gapdh cDNA, and against $18 S$ [gene encoding ribosomal RNA 18S] (QuantumRNA Internal Standards, Ambion, Austin, TX) within the same sample. For subsequent work, the cDNA concentration of the gene of interest was normalized against the concentration of $A c t b$ cDNA within the same sample, and the results were finally expressed as percentage of increase versus the control (untreated neurons or neurons treated with vehicle). In each experiment, the average values of triplicate samples were used for each data point.

2.3.1 qPCR Primers-The following primers (and acquisition temperatures) were used for qPCR: Syt $1\left(70^{\circ} \mathrm{C}\right)$ forward (5'-CACCGTGGGCCTTAATTGC-3'), reverse (5'-

TGTTAATGGCGTTCTTCCCTC-3'); Actb $\left(82^{\circ} \mathrm{C}\right)$ forward (5'-

TCATGAAGTGTGACGTTGACATCCGT-3'), reverse (5'-

CCTAGAAGCATTTGCGGTGCACGATG-3'); $\operatorname{Gapdh}\left(77^{\circ} \mathrm{C}\right)$ forward $\left(5^{\prime}\right.$ -

AACTTTGGCATTGTGGAAGG-3'), reverse (5'-ACACATTGGGGGTAGGAACA-3'); Snap $25\left(75^{\circ} \mathrm{C}\right)$ forward (5' -CAACTGGAACGCATTGAGGAA-3'), reverse (5'-

GGCCACTACTCCATCCTGATTAT-3'); Stxla $\left(77^{\circ} \mathrm{C}\right)$ forward (5'-

TCCAAGCTAAAGAGCATTGAGC-3'), reverse (5'-

GGCGTTGTACTCGGACATGA-3'); Sypl $\left(77^{\circ} \mathrm{C}\right)$ forward (5'- 
GAGAGAACAACAAAGGGCCAA-3'), reverse (5'-CGGCACATAGGCATCTCCT-3'); Vampl $\left(72^{\circ} \mathrm{C}\right)$ forward $\left(5^{\prime}\right.$-AGCATCACAATTTGAGAGCAGT-3'), reverse (5' GATGGCACAGATAGCTCCCAG-3'); $\operatorname{Vamp} 2\left(76^{\circ} \mathrm{C}\right)$ forward $\left(5^{\prime}\right.$ GCTGGATGACCGTGCAGAT-3'), reverse (5'-GATGGCGCAGATCACTCCC-3').

\subsection{Immunoblotting}

Relative protein abundance was determined by immunoblotting, as previously described (Jia et al., 2005). Cellular fractions (40-100 mg of protein) were isolated with the NE-PER Nuclear and Cytoplasmic Extraction Reagents (Pierce Biotechnology, Rockford, IL) and incubated with the following antibodies: rabbit polyclonal anti-Syt1 (1:1500, Synaptic Systems), rabbit polyclonal anti-HSF1 (1:500, Cell Signaling Technology, Danvers, MA), rabbit polyclonal anti-phosphorylated HSF1 (pHSF1, 1:4000, Enzo Life Sciences, Farmingdale, NY), rabbit polyclonal anti-VAMP1 (1:500, Synaptic Systems), mouse monoclonal anti-VAMP2 (1:2000, Synaptic Systems), mouse monoclonal anti- $\alpha$-tubulin (1:5000, clone DM1A, Sigma-Aldrich), and rabbit polyclonal anti-eIF4E (1:2500, Cell Signaling Technology). Images were acquired with a refrigerated Chemi 410 CCD camera, the Biospectrum imaging system (UVP, Upland, CA), and the VisionWorks LS software (UVP). Digital images were quantified with ImageJ 1.36b (NIH, Bethesda, MD), with gel lanes selected and their signals transformed into peaks. The area under each peak (gray value) was transformed into an optical density (OD) value using the function: $O D=\log _{10}$ (255 / (255 - gray value)). The OD values were normalized to the $\alpha$-tubulin or eIF4E internal standards to compensate for variations in protein loading and transfer.

\subsection{RNA interference experiments}

RNA interference experiments were performed with pre-synthesized small interference RNA (siRNA), consisting of a pool of 3 target-specific 20-25 nucleotide siRNAs designed to knock down the expression of a particular gene. Cultured cortical neurons were transfected on DIV 7 with $H s f l$ siRNA or control siRNAs (Santa Cruz Biotechnology, Santa Cruz, CA). Transfection was performed with TransFectin (Bio-Rad) as follows: siRNA $(0.33 \mu \mathrm{g})$ was added to OPTI-MEM (50 $\mu \mathrm{l}$; Invitrogen) for $5 \mathrm{~min}$ and then combined with a mixture of TransFectin $(2.6 \mu \mathrm{l})$ and OPTI-MEM $(50 \mu \mathrm{l})$ for an additional $20 \mathrm{~min}$. The culture medium was removed and replaced with $100 \mu \mathrm{l}$ of transfection medium and the neurons were incubated for $1 \mathrm{~h}$ at $37^{\circ} \mathrm{C}$. Cells were washed once and the transfection medium replaced with conditioned medium; neurons were maintained for another $24 \mathrm{~h}$ prior to ethanol or heat treatment. Control experiments were performed with a scrambled 20-25 nucleotide siRNA (control siRNA), which does not degrade any known mRNA (Pignataro et al., 2007).

\subsection{Constitutively active and inactive heat shock factor 1 (Hsf1) constructs}

We made use of a constitutively active form of HSF1 (Hsfl-act, BH-S), as well as a dominant-negative mutant form of HSF1 (Hsfl-inact, AV-ST). Hsfl-act has a long deletion of amino acids 203-315 in the regulatory domain of HSF1 (Zuo et al., 1995), while the dominant-negative mutant form of HSF1 has a deletion of amino acids 453-523 located in the transcription activation domain (Zuo et al., 1995). Both constructs were generated by Dr. Richard Voellmy (University of Miami) and cloned into pcDNA3.1+ (Invitrogen). Transfections were performed as described above, with $1 \mu \mathrm{g}$ of DNA and $9 \mu \mathrm{L}$ of nupherin (Enzo Life Sciences), and sister cultures were transfected with the empty pcDNA3.1+ vector as controls. 


\subsection{Statistical, Microscopy and Genome Analyses}

Briefly, the qPCR and immunoblot data were analyzed by 1-way ANOVA and compared with control samples using Dunnett's multiple-comparison post-hoc test. All data are presented as mean \pm SEM and the details of the statistical analysis are also included in the appropriate figure legends. In all cases in which immunoblots are shown, the blot is representative of at least three experiments with similar results.

The analysis of Syt1 immunoreactive puncta was performed by standard methods (Carpenter-Hyland et al., 2004) using ImageJ 1.36b software. Grayscale 8-bit calibratedimages ( 0.8 to $1 \mathrm{~mm}$ optical section) were manually adjusted for threshold, and the area and number of Syt1 clusters present along neurites was calculated. Particles smaller than $\sim 0.01$ $\mu \mathrm{m}^{2}$ were not considered to be Syt1-positive puncta and were discarded from the analysis.

For all genes analyzed, mouse genomic DNA sequence was obtained from the National Center for Biotechnology Information (NCBI; NIH) database. DNA sequence analyses were performed using Vector NTI (Invitrogen) and putative ARE sites were designated as those containing the ARE motif (tCTGcGTCtCt, uppercase letters indicate absolute conservation) anywhere between $2 \mathrm{~kb}$ upstream of the ATG and the 3'-untranslated region.

\section{Results}

\subsection{Alcohol up-regulates Syt1 mRNA and protein expression in cortical neurons}

Our initial experiments confirmed earlier findings from microarray work that Sytl is an alcohol- and heat stress-responsive gene (Pignataro et al., 2007). We found that exposure of cultured mouse cortical neurons to $60 \mathrm{mM}$ ethanol for 1 hour produced a robust $(58 \pm 3 \%)$ stimulation of Syt 1 mRNA levels, which was reproduced by a $42^{\circ} \mathrm{C}$ heat shock treatment for one hour (Fig. 1A). The results of ethanol treatment on Syt 1 induction were not significantly different when Sytl expression was normalized to three different internal standards, Actb, Gapdh and $18 S$ [gene encoding ribosomal RNA 18S] (data not shown). Both alcohol and heat shock treatments also increased Syt1 protein levels (Fig. 1B).

The ethanol concentration used in these initial experiments $(60 \mathrm{mM})$ is high, but relevant to human exposure, as chronic alcoholics may routinely tolerate extremely high blood alcohol concentrations of $100 \mathrm{mM}$ or above (Urso et al., 1981). Nevertheless, we wanted to examine the effects of ethanol exposure at concentrations more relevant to social intoxication, so we performed a concentration-response analysis for up-regulation of Syt 1 mRNA levels. We found that the effect of ethanol (E) on Syt 1 mRNA levels was concentration-dependent (Fig. $2 \mathrm{~A}$ ), with a sensitivity threshold of $20 \mathrm{mM}$ and half-maximal activation at $50 \pm 1 \mathrm{mM}$; the ethanol effect saturated between 100 and $200 \mathrm{mM}$. Surprisingly, even these very high ethanol concentrations were not toxic to the neurons, with short-term exposure to ethanol concentrations $>100 \mathrm{mM}$ resulting in only a modest increase in apoptosis, as previously reported (Pignataro et al., 2007). The time-course of the activation of Sytl transcription by $60 \mathrm{mM}$ ethanol was surprisingly rapid, increasing mRNA levels significantly within 30 minutes of exposure (Fig. 2B). Syt1 up-regulation continued to increase during 8 hours of exposure to $60 \mathrm{mM}$ ethanol but then declined, although not to baseline levels, following 24 hours of continuous exposure. Ethanol also increased Syt1 protein, although this effect was delayed relative to the effect on Sytl mRNA, with a significant rise in protein occurring after 8-12 hours of exposure (Fig. 2C, D).

\subsection{Alcohol alters the distribution of Syt1 immunoreactivity in cortical neurons}

To visualize changes in Syt1 protein localization after ethanol exposure, we performed immunocytochemistry and confocal microscopy on the cortical cultures. Syt1 protein is 
embedded in the synaptic vesicle membrane and, as expected, in both control and ethanoltreated neurons we observed punctate clusters of Syt1-positive staining along the neurites (Fig. 3A). Ethanol exposure affected the distribution of Syt1 protein, as observed in the representative inset figures. Quantification revealed that ethanol increased the number of identifiable Syt1-positive puncta per $100 \mu \mathrm{m}$ of neurite length by 2.2 -fold, and also increased the average area of the puncta by 1.6-fold (Fig. 3B). Both findings are consistent with an increase in the level of Syt1 protein in these neurons.

\subsection{Alcohol activates HSF1 to induce Syt1 expression in cortical neurons}

It has been reported that ethanol and heat up-regulate the Gabra4 gene via activation of the transcription factor, heat shock factor 1 (HSF1; Pignataro et al., 2007). HSF1 transcriptional activation is a multi-step process, requiring translocation to the nucleus, followed by trimerization and inducible hyperphosphorylation (Cotto et al., 1996). It has been demonstrated in primary cortical neuron culture that ethanol and heat shock can induce HSF1 nuclear translocation (Pignataro et al., 2007). To investigate whether HSF1 plays a similar role in Syt1 induction by ethanol, we first assessed the effects of ethanol and heat on HSF1 phosphorylation (pHSF1) and then altered HSF1 protein levels to affect Syt 1 induction by ethanol. We found that 2 hours of $60 \mathrm{mM}$ ethanol or heat treatment increased the phosphorylation of HSF1 protein, as shown in the inset in Fig 4A. The data shows that the proportion of pHSF1 relative to the total expression of HSF1 is increased significantly by ethanol and heat, suggesting activation of this transcription factor (Fig 4A). Since previous work showed that a 24 hour treatment of cortical neurons with $H s f l$ siRNA produced a $>70 \%$ decrease in Hsfl mRNA and HSF1 protein levels (Pignataro et al., 2007), we used the same HSF1 knock-down protocol here, and observed a reduction in Sytl gene induction in response to ethanol exposure (Fig. 4B).

To confirm the role of HSF1 in mediating Sytl gene induction, we then transfected cortical neurons with a constitutively active $H s f l$ construct ( $H s f l$-act), which is known to directly induce heat shock protein $(\mathrm{Hsp})$ gene transcription in the absence of heat stress (AcquaahMensah et al., 2001). This construct increased Syt1 gene expression to a level similar to that seen after 1 hour of ethanol exposure (Fig. 4C). Conversely, a dominant-negative Hsf 1 construct ( $H s f l$-inact), which prevents the induction of $H s p$ expression (Acquaah-Mensah et al., 2001), abolished the effect of ethanol exposure on Syt1 mRNA levels (Fig. 4C). This confirmed that activated HSF1 is both sufficient for Syt 1 induction, and necessary for the up-regulation of this gene by ethanol. In the case of Gabra4 induction by ethanol, it was found that the activated HSF1 binds to the alcohol response element (ARE; Pignataro et al., 2007) identified in a subset of alcohol-responsive genes (Kwon et al., 2004; Pignataro et al., 2007). The Sytl gene (Gene ID: 20979) contains multiple putative ARE sequences, including multiple repeat ARE-like sequences located within the promoter region and in the first and second introns (Fig. 5C).

\subsection{Alcohol induces a subset of genes encoding synaptic vesicle proteins in cortical neurons}

To identify whether Sytl induction by ethanol and heat stress is a specific response, we examined the effects of ethanol exposure on genes that code for other proteins intimately involved in synaptic vesicle fusion. We found that the ability of ethanol and heat stress to induce Syt 1 gene expression is replicated among a subset of SNARE complex genes (Fig. $5 \mathrm{~A})$. We observed that ethanol $(60 \mathrm{mM}, 1 \mathrm{~h})$ and heat shock treatment also up-regulate SNAP-25 (Snap25) and VAMP2 (Vamp2) gene expression, while syntaxin-1A (Stxla), synaptophysin 1 (Syp1), and VAMP1 (Vamp1) were not significantly altered by either treatment. Consistent with these changes in mRNA levels, ethanol and heat shock also increase VAMP2 protein levels, without affecting VAMP1 expression (Fig 5B). Sequence 
analysis of Vampl (Gene ID: 22317) and Vamp2 (Gene ID: 22318) revealed two putative ARE sequences in each gene, but the sequences in Vampl are located far downstream in the 3'-untranslated region of the gene, while the candidate ARE in Vamp2 are located in the second intron, as in Gabra4 (Fig 5C). These findings demonstrate that the effects of ethanol are specific to particular synaptic machinery proteins, and not a generalized phenomenon across all proteins expressed at the synaptic terminal.

\section{Discussion}

In the last decade several studies have demonstrated that ethanol can alter neurotransmitter release, especially that of GABA, in the central nervous system (Siggins et al., 2005; Roberto et al., 2006; Weiner and Valenzuela, 2006). The mechanisms of these effects have received limited attention, but in the cerebellum, the increased release of GABA on to granule cells appears to be secondary to an increase in Golgi cell firing rate (Carta et al., 2004). The effects of ethanol on the synaptic vesicle fusion machinery that governs neurotransmitter release have remained largely unstudied, but a recent microarray screen identified a number of ARGs that encode synaptic proteins. In this way, Sytl was first suggested to be an ARG that is also sensitive to heat shock (Pignataro et al., 2007), but microarray studies can sometimes produce either false negative or false positive results. Our detailed analysis using qPCR confirmed that acute exposure to ethanol increases Syt 1 expression in cortical neurons by activating the transcription factor HSF1. In addition, the effects of acute ethanol exposure on Sytl transcription are time-dependent, with mRNA levels peaking around 6-8 hours, and then fading between 8 and 24 hours after the onset of exposure. The increase in Syt1 protein during acute alcohol exposure might be expected to result in changes in transmitter release from these neurons, and this possibility is currently being investigated.

There are few, if any, comparable studies available on the effects of acute alcohol in vitro or short-term drinking in vivo on synaptic gene expression. A post-mortem human microarray study found that Sytl gene expression was decreased in the nucleus accumbens, but not the frontal cortex, of alcoholic brains compared to control cases (Flatscher-Bader et al., 2005). We have not yet examined the effects of longer-term exposure to alcohol on Syt1 expression in the cortical neurons, so it is very difficult to compare our data with those in human brain.

Little information is currently available on Syt1 expression from in vivo alcohol drinking studies. Syt1 protein levels were reduced in the cerebellum of rat pups exposed to binge-like ethanol by oral-gastric intubation, but remained unchanged in the medial septum/diagonal band, cerebral cortex, hippocampus and brain stem (Hsiao et al., 2002). A study of rats genetically selected for either alcohol self-administration preference (AA) or alcohol avoidance (ANA), showed a decrease in Sytl gene expression in the frontal cortex of the AA rats (Worst et al., 2005). The discrepancies among these sets of data and our own work are likely to stem from variations in model systems, brain regions of interest, and alcohol exposure paradigms. Future efforts in our laboratory will therefore be aimed at studying the effects on synaptic gene expression of longer term exposure to alcohol in the cortical neurons, and in animals engaged in short-term drinking.

Alcohol exposure increased Syt1 immunoreactivity in the cortical neurons, with the Syt1 immunoreactivity seen as distinct puncta on long neurites and around the cell somata, which is a characteristic of synaptic regions (Matthew et al., 1981; Gitler et al., 2004). Of note, a similar study using chronic (4 days) ethanol treatment of primary hippocampal cultures also revealed an increase in the size and density of synapsin- and synaptophysin-positive clusters (Carpenter-Hyland et al., 2004). Our findings that ethanol increases Syt1-labeled puncta number and size are completely novel, but we cannot as yet determine whether these 
changes in immunoreactivity represent an increase in the number of Syt1 protein molecules per synaptic vesicle, larger numbers or aggregations of synaptic vesicles, or increases in synaptic vesicle size.

Sytl shares its alcohol and heat stress-responsive properties with Gabra4, which is induced by ethanol when activated HSF1 binds to a specific sequence within the second intron, a sequence we have termed the ARE (Pignataro et al., 2007). The ARE is an 11 base pair cisregulatory element (tCTGcGTCtCt, uppercase letters indicate absolute conservation) that was first identified in a subset of ARG in C. elegans (Kwon et al., 2004; Pignataro et al., 2007). The element is a consensus-binding site for HSF1, though its sequence is distinct from the classical heat shock element (HSE; Pignataro et al., 2007). The Syt1 gene contains multiple candidate ARE sequences, and neuronal transfection of a constitutively active $H s f l$ construct (Hsfl-act) induces $S y t l$ in a similar manner to ethanol exposure. $H s f l$-act is known to induce heat shock protein genes (Hsps) in the absence of heat stress (Acquaah-Mensah et al., 2001). Conversely, a dominant-negative $H s f l$ construct (Hsfl-inact), which cannot induce heat shock proteins gene expression (Acquaah-Mensah et al., 2001), abolished the effect of ethanol exposure on Syt1. As transcription of Gabra4 and the Hsps are both dependent on HSF1, the results of these experiments strongly suggest that Syt 1 induction by alcohol is mediated via the heat shock pathway, and that alcohol acts upstream of HSF1 activation. Since the ARE is a highly conserved sequence, it is very likely that HSF1 binds to many of these sites throughout the Syt1 gene. This raises the interesting question of whether only some of the HSF1-bound ARE sites are able to promote Syt1 transcription. Future work will address these potential position-dependent effects of the ARE.

Syt1 does not act alone in promoting neurotransmitter release, participating with several other key synaptic proteins to mediate vesicle fusion. Given the large variation in the effects of alcohol on neurotransmitter release that is observed across the brain, it is possible that alcohol differentially regulates some of these other synaptic terminal proteins. We were therefore very interested in specifically assessing whether genes that encode other key vesicle fusion machinery proteins are regulated by ethanol and heat shock. In the microarray experiment of Pignataro et al. (2007), for example, Vamp2 and Vamp8 (but not Vamp1) were shown to be sensitive to alcohol and heat shock. In our qPCR experiments, we found that ethanol and heat shock also induced Snap25 and Vamp2, while mRNA for synaptophysin 1 (Syp1), syntaxin-1A (Stxla) and Vampl remained unchanged under both treatment conditions.

When considered in this light, ethanol and heat stress induction of Vamp2, but not Vampl, is particularly interesting. These two genes encode distinct VAMP isoforms and are differentially expressed in the CNS (Nystuen et al., 2007). Vamp2 is expressed throughout the mouse brain, particularly in the cortex, whereas Vampl predominates in regions of the diencephalon and midbrain. Though both genes contain potential ARE sequences, in Vamp1 the sequences are all located far downstream in the 3'-untranslated region of the gene, while the candidate ARE in Vamp2 are located in the second intron, as in Gabra4. The selective induction of Vamp2, but not Vamp1, by ethanol, is therefore also likely to be dependent on the location of the ARE sequence.

The biological implications of these findings in terms of the functional consequences of Syt1 regulation by ethanol are unclear. Flatscher-Bader et al. (2005), noted a decrease in Syt1 gene expression in the nucleus accumbens of alcoholics, consistent with studies showing a down-regulation in the mesolimbic dopamine system after chronic alcohol consumption. Our present work shows that acute ethanol induces Syt 1 transcription, and it is well established that Syt1 over-expression in mouse hippocampal neurons increases the probability of neurotransmitter release (Han et al., 2004). This suggests a potential 
molecular mechanism by which vesicle fusion is increased to stabilize increased neurotransmitter release during intermediate alcohol exposures, over several hours. The specificity of this effect among variable synapses and cell types (GABA vs. glutamate; interneuron $v s$. principal cells) is obviously a key issue and remains to be investigated. Future experiments will be aimed at determining, using molecular and electrophysiological approaches, whether alcohol is able to regulate Syt1 and other key synaptic proteins universally or whether this effect is restricted to specific neurotransmitter profiles, neuronal cell types or brain regions.

\section{Acknowledgments}

We thank H.D Durham (McGill University, Montreal, Quebec, Canada) for providing the Hsfl constructs and R. Voellmy (University of Miami, Miami, FL) for permission to use them. This work was supported by NIH grants to N.L.H.

\section{Abbreviations}

$\begin{array}{ll}\text { ARE } & \text { alcohol response element } \\ \text { ARG } & \text { alcohol-responsive gene } \\ \text { DIV } & \text { days in vitro } \\ \text { GABA } & \gamma \text {-aminobutyric acid } \\ \text { Hsf1, HSF1 } & \text { heat shock factor 1 } \\ \text { Hsp } & \text { heat shock protein } \\ \text { OD } & \text { optical density } \\ \text { qPCR } & \text { quantitative polymerase chain reaction } \\ \text { siRNA } & \text { small interfering RNA } \\ \text { SNAP-25 } & \text { synaptosomal-associated protein 25 } \\ \text { SNARE } & \text { soluble NSF attachment protein receptor } \\ \text { Stx1a } & \text { syntaxin 1a } \\ \text { Syt1 } & \text { synaptotagmin 1 } \\ \text { Syp1 } & \text { synaptophysin 1 } \\ \text { VAMP } & \text { vesicle-associated membrane protein }\end{array}$

\section{References}

Acquaah-Mensah GK, Leslie SW, Kehrer JP. Acute exposure of cerebellar granule neurons to ethanol suppresses stress-activated protein kinase-1 and concomitantly induces AP-1. Toxicol Appl Pharmacol. 2001; 175:10-18. [PubMed: 11509022]

Banker, G.; Goslin, K., editors. Culturing nerve cells. Cambridge: MIT Press; 1991.

Brose N, Petrenko AG, Sudhof TC, Jahn R. Synaptotagmin: a calcium sensor on the synaptic vesicle surface. Science. 1992; 256:1021-1025. [PubMed: 1589771]

Carpenter-Hyland EP, Woodward JJ, Chandler LJ. Chronic ethanol induces synaptic but not extrasynaptic targeting of NMDA receptors. J Neurosci. 2004; 24:7859-7868. [PubMed: 15356198]

Carta M, Ariwodola OJ, Weiner JL, Valenzuela CF. Alcohol potently inhibits the kainate receptordependent excitatory drive of hippocampal interneurons. Proc Natl Acad Sci U S A. 2003; 100:6813-6818. [PubMed: 12732711] 
Carta M, Mameli M, Valenzuela CF. Alcohol enhances GABAergic transmission to cerebellar granule cells via an increase in Golgi cell excitability. J Neurosci. 2004; 24:3746-3751. [PubMed: 15084654]

Cotto JJ, Kline M, Morimoto RI. Activation of heat shock factor 1 DNA binding precedes stressinduced serine phosphorylation. J Biol Chem. 1996; 271:3355-3358. [PubMed: 8631933]

Crowder TL, Weiner JL. Functional characterization of kainate receptors in the rat nucleus accumbens core region. J Neurophysiol. 2002; 88:41-48. [PubMed: 12091531]

Flatscher-Bader T, van der Brug M, Hwang JW, Gochee PA, Matsumoto I, Niwa S, Wilce PA. Alcohol-responsive genes in the frontal cortex and nucleus accumbens of human alcoholics. $\mathrm{J}$ Neurochem. 2005; 93:359-370. [PubMed: 15816859]

Geppert M, Goda Y, Hammer RE, Li C, Rosahl TW, Stevens CF, Sudhof TC. Synaptotagmin I: a major $\mathrm{Ca} 2+$ sensor for transmitter release at a central synapse. Cell. 1994; 79:717-727. [PubMed: 7954835]

Gitler D, Xu Y, Kao HT, Lin D, Lim S, Feng J, Greengard P, Augustine GJ. Molecular determinants of synapsin targeting to presynaptic terminals. J Neurosci. 2004; 24:3711-3720. [PubMed: 15071120]

Han W, Rhee JS, Maximov A, Lao Y, Mashimo T, Rosenmund C, Sudhof TC. N-glycosylation is essential for vesicular targeting of synaptotagmin 1. Neuron. 2004; 41:85-99. [PubMed: 14715137]

Harris RA. Ethanol actions on multiple ion channels: which are important? Alcohol Clin Exp Res. 1999; 23:1563-1570. [PubMed: 10549986]

Hsiao SH, Parrish AR, Nahm SS, Abbott LC, McCool BA, Frye GD. Effects of early postnatal ethanol intubation on GABAergic synaptic proteins. Brain Res Dev Brain Res. 2002; 138:177-185.

Huettner JE, Baughman RW. Primary culture of identified neurons from the visual cortex of postnatal rats. J Neurosci. 1986; 6:3044-3060. [PubMed: 3760948]

Jia F, Pignataro L, Schofield CM, Yue M, Harrison NL, Goldstein PA. An extrasynaptic GABAA receptor mediates tonic inhibition in thalamic VB neurons. J Neurophysiol. 2005; 94:4491-4501. [PubMed: 16162835]

Kwon JY, Hong M, Choi MS, Kang S, Duke K, Kim S, Lee S, Lee J. Ethanol-response genes and their regulation analyzed by a microarray and comparative genomic approach in the nematode Caenorhabditis elegans. Genomics. 2004; 83:600-614. [PubMed: 15028283]

Lewohl JM, Wang L, Miles MF, Zhang L, Dodd PR, Harris RA. Gene expression in human alcoholism: microarray analysis of frontal cortex. Alcohol Clin Exp Res. 2000; 24:1873-1882. [PubMed: 11141048]

Lovinger DM. Alcohols and neurotransmitter gated ion channels: past, present and future. Naunyn Schmiedebergs Arch Pharmacol. 1997; 356:267-282. [PubMed: 9303562]

Ma L, Song L, Radoi GE, Harrison NL. Transcriptional regulation of the mouse gene encoding the alpha-4 subunit of the GABAA receptor. J Biol Chem. 2004; 279:40451-40461. [PubMed: 15265862]

Maldve RE, Chen X, Zhang TA, Morrisett RA. Ethanol selectively inhibits enhanced vesicular release at excitatory synapses: real-time visualization in intact hippocampal slices. Alcohol Clin Exp Res. 2004; 28:143-152. [PubMed: 14745313]

Martens S, Kozlov MM, McMahon HT. How synaptotagmin promotes membrane fusion. Science. 2007; 316:1205-1208. [PubMed: 17478680]

Matthew WD, Tsavaler L, Reichardt LF. Identification of a synaptic vesicle-specific membrane protein with a wide distribution in neuronal and neurosecretory tissue. J Cell Biol. 1981; 91:257269. [PubMed: 7298720]

Mulligan MK, Ponomarev I, Hitzemann RJ, Belknap JK, Tabakoff B, Harris RA, Crabbe JC, Blednov YA, Grahame NJ, Phillips TJ, Finn DA, Hoffman PL, Iyer VR, Koob GF, Bergeson SE. Toward understanding the genetics of alcohol drinking through transcriptome meta-analysis. Proc Natl Acad Sci U S A. 2006; 103:6368-6373. [PubMed: 16618939]

Nystuen AM, Schwendinger JK, Sachs AJ, Yang AW, Haider NB. A null mutation in VAMP1/ synaptobrevin is associated with neurological defects and prewean mortality in the lethal-wasting mouse mutant. Neurogenetics. 2007; 8:1-10. [PubMed: 17102983] 
Pignataro L, Miller AN, Ma L, Midha S, Protiva P, Herrera DG, Harrison NL. Alcohol regulates gene expression in neurons via activation of heat shock factor 1. J Neurosci. 2007; 27:12957-12966. [PubMed: 18032669]

Roberto M, Madamba SG, Moore SD, Tallent MK, Siggins GR. Ethanol increases GABAergic transmission at both pre- and postsynaptic sites in rat central amygdala neurons. Proc Natl Acad Sci U S A. 2003; 100:2053-2058. [PubMed: 12566570]

Roberto M, Treistman SN, Pietrzykowski AZ, Weiner J, Galindo R, Mameli M, Valenzuela F, Zhu PJ, Lovinger D, Zhang TA, Hendricson AH, Morrisett R, Siggins GR. Actions of acute and chronic ethanol on presynaptic terminals. Alcohol Clin Exp Res. 2006; 30:222-232. [PubMed: 16441271]

Siggins GR, Roberto M, Nie Z. The tipsy terminal: presynaptic effects of ethanol. Pharmacol Ther. 2005; 107:80-98. [PubMed: 15963352]

Urso T, Gavaler JS, Van Thiel DH. Blood ethanol levels in sober alcohol users seen in an emergency room. Life Sci. 1981; 28:1053-1056. [PubMed: 7219065]

Valtorta F, Pennuto M, Bonanomi D, Benfenati F. Synaptophysin: leading actor or walk-on role in synaptic vesicle exocytosis? Bioessays. 2004; 26:445-453. [PubMed: 15057942]

Weiner JL, Valenzuela CF. Ethanol modulation of GABAergic transmission: the view from the slice. Pharmacol Ther. 2006; 111:533-554. [PubMed: 16427127]

Worst TJ, Tan JC, Robertson DJ, Freeman WM, Hyytia P, Kiianmaa K, Vrana KE. Transcriptome analysis of frontal cortex in alcohol-preferring and nonpreferring rats. J Neurosci Res. 2005; 80:529-538. [PubMed: 15846778]

Xu J, Mashimo T, Sudhof TC. Synaptotagmin-1, -2, and -9: Ca(2+) sensors for fast release that specify distinct presynaptic properties in subsets of neurons. Neuron. 2007; 54:567-581. [PubMed: 17521570]

Ziskind-Conhaim L, Gao BX, Hinckley C. Ethanol dual modulatory actions on spontaneous postsynaptic currents in spinal motoneurons. J Neurophysiol. 2003; 89:806-813. [PubMed: 12574458]

Zuo J, Rungger D, Voellmy R. Multiple layers of regulation of human heat shock transcription factor 1. Mol Cell Biol. 1995; 15:4319-4330. [PubMed: 7623826] 


\section{Research Highlights}

Acute alcohol increases Syt1 gene and protein expression in mouse cortical neurons.

Syt 1 gene induction by ethanol requires the activation of heat shock factor 1 (HSF1).

Alcohol regulates the gene expression of other core synaptic vesicle fusion proteins. 


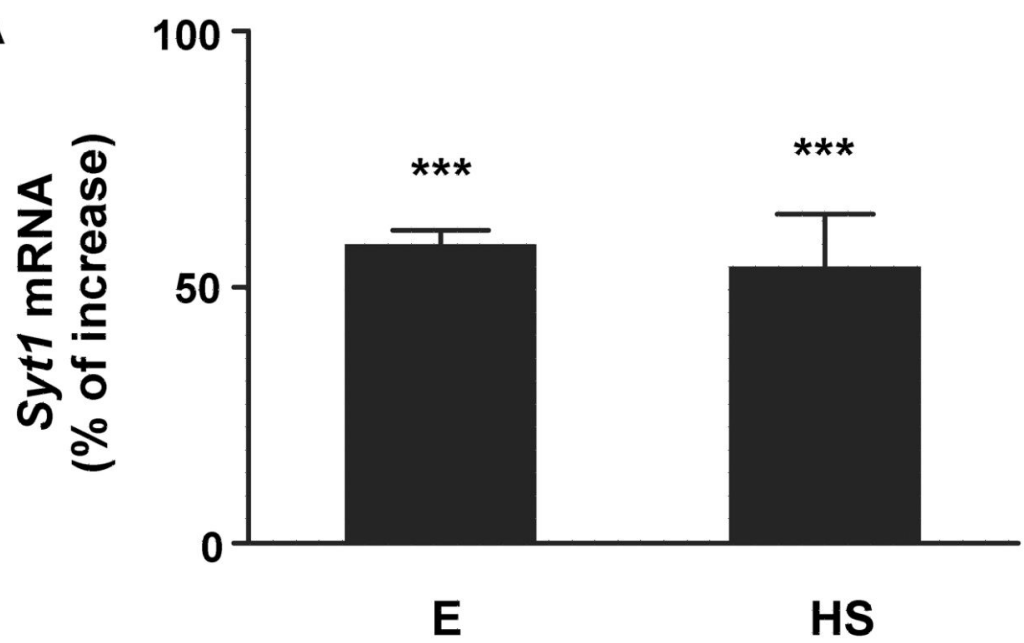

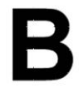

\section{KDa}

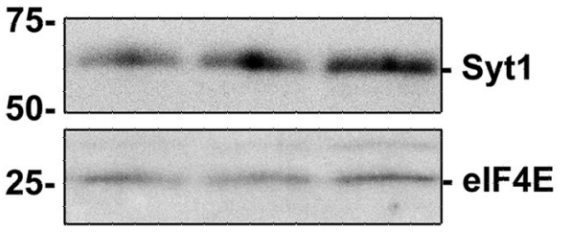

C E HS

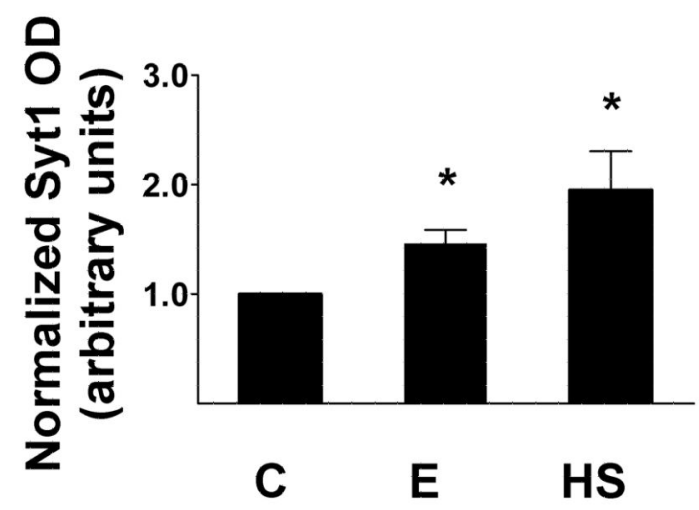

Figure 1. Ethanol and heat shock alter Syt1 expression in cortical neurons

$\boldsymbol{A}$, Increase in Syt $1 \mathrm{mRNA}$ expression after treatment for 1 hour with $60 \mathrm{mM}$ ethanol (E) or $42^{\circ} \mathrm{C}$ heat shock (HS), as measured by qPCR. The data were normalized to Actb cDNA, and compared with control samples treated with vehicle using 1-way ANOVA and Dunnett's multiple-comparison post-hoc test $(\mathrm{n} \geq 6 ; \mathrm{F}=63.82$, $\mathrm{df}=31)$. All data are mean $\pm \mathrm{SEM}$ (*** significantly different at the level of $p<0.001$ ).

$\boldsymbol{B}$, Increase in Syt1 protein after treatment with ethanol or heat shock. The graph shows the relative abundance of Syt1 protein in neurons exposed for 2 hours to $60 \mathrm{mM}$ ethanol (E), a $42^{\circ} \mathrm{C}$ heat shock (HS) or vehicle control (C). The bar graph represents normalized optical density (OD) relative to the control sample. Ethanol and heat shock data were compared with control by 1-way ANOVA, with Dunnett's multiple-comparison post-hoc test ( $\mathrm{n} \geq 3$; F $=5.427, \mathrm{df}=14)$. All data are mean \pm SEM (* significantly different at the level of $p<$ $0.05)$. 

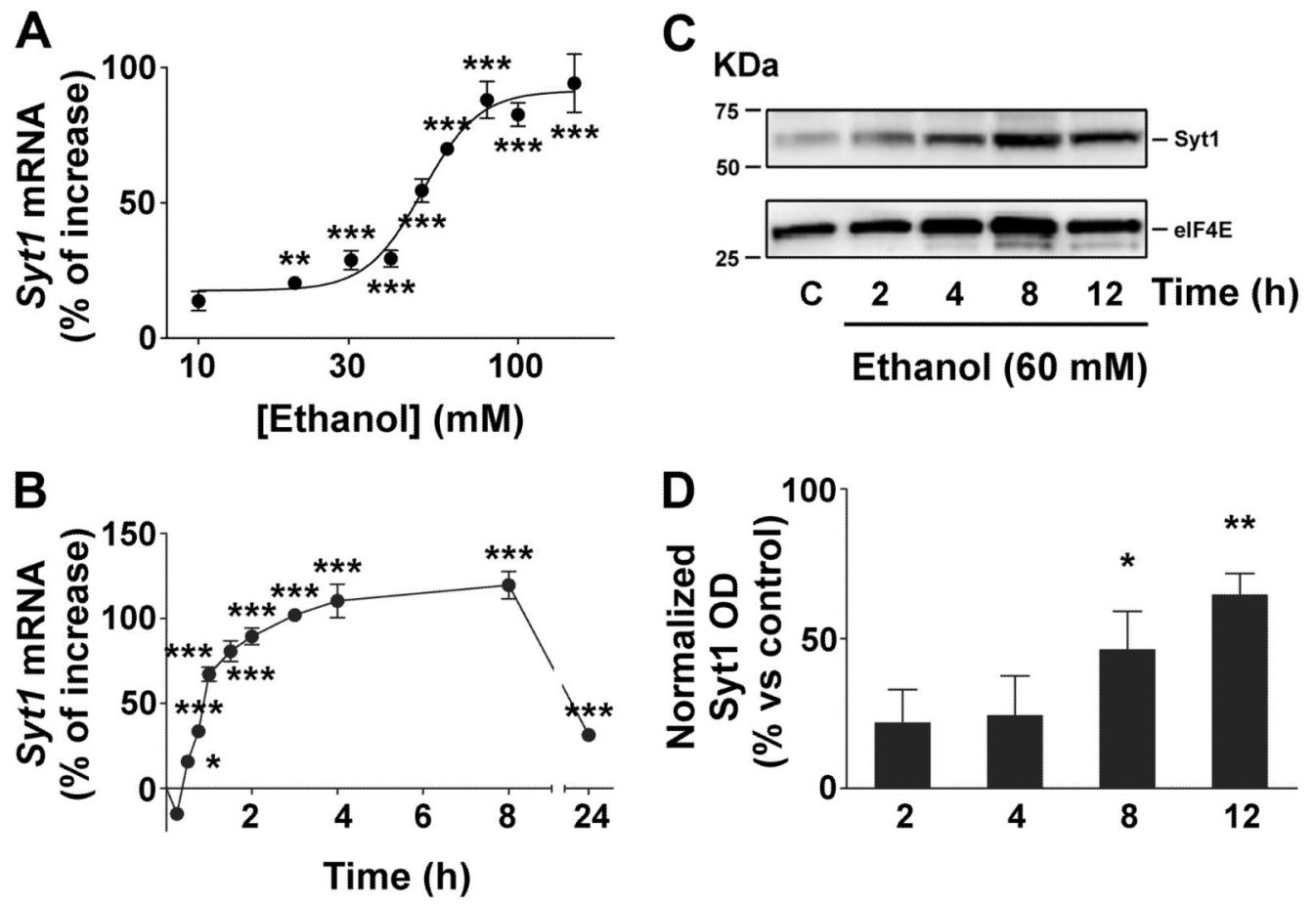

Figure 2. Ethanol increases Syt1 mRNA and protein expression

$\boldsymbol{A}$, Increase in Syt1 mRNA expression after 1 hour treatment with different concentrations of ethanol, as measured by qPCR. The data were normalized to Actb cDNA, and the ethanolexposed samples were compared with control samples using repeated-measures ANOVA and Dunnett's multiple-comparison post-hoc test ( $\mathrm{n} \geq 6 ; \mathrm{F}=53.54$, $\mathrm{df}=79$ ). The halfmaximal activation of Syt 1 was calculated as $50 \pm 1 \mathrm{mM}$, and the ethanol sensitivity threshold was found to be $20 \mathrm{mM}$ ( $p<0.001$ ). This threshold is the lowest ethanol concentration that significantly increased $S y t 1$ expression above the control value and was obtained by analyzing the tail of the concentration-response curve with one-tailed unpaired $t$ test. All data are mean \pm SEM (** significantly different at the level of $p<0.01, * * * p<$ 0.001).

$\boldsymbol{B}$, Increase in Syt $1 \mathrm{mRNA}$ expression after $60 \mathrm{mM}$ ethanol exposure over time, as measured by qPCR. The data were normalized to Actb cDNA, and the ethanol-exposed samples were compared with control samples using 1-way ANOVA and Dunnett's multiple-comparison post-hoc test $(\mathrm{n} \geq 6 ; \mathrm{F}=103.01, \mathrm{df}=108)$. All data are mean \pm SEM (* significantly different at the level of $p<0.05, * * * p<0.001)$.

$\boldsymbol{C}$, Increase in Syt1 protein after treatment with ethanol over extended periods of time. The graph shows the relative abundance of Syt1 protein in neurons treated with $60 \mathrm{mM}$ ethanol for different time periods of exposure, or vehicle control (C).

$\boldsymbol{D}$, Increase in Syt1 protein after treatment with $60 \mathrm{mM}$ ethanol for different time periods of exposure. The bar graph represents normalized Syt1 optical density (OD) for each ethanolexposed sample relative to the control sample. The data were compared with control values by 1-way ANOVA with Dunnett's multiple-comparison post-hoc test ( $\mathrm{n} \geq 3 ; \mathrm{F}=6.136$, df = 19). All data are mean $\pm \operatorname{SEM}$ (* significantly different at the level of $p<0.05, * * p<0.01$ ). 

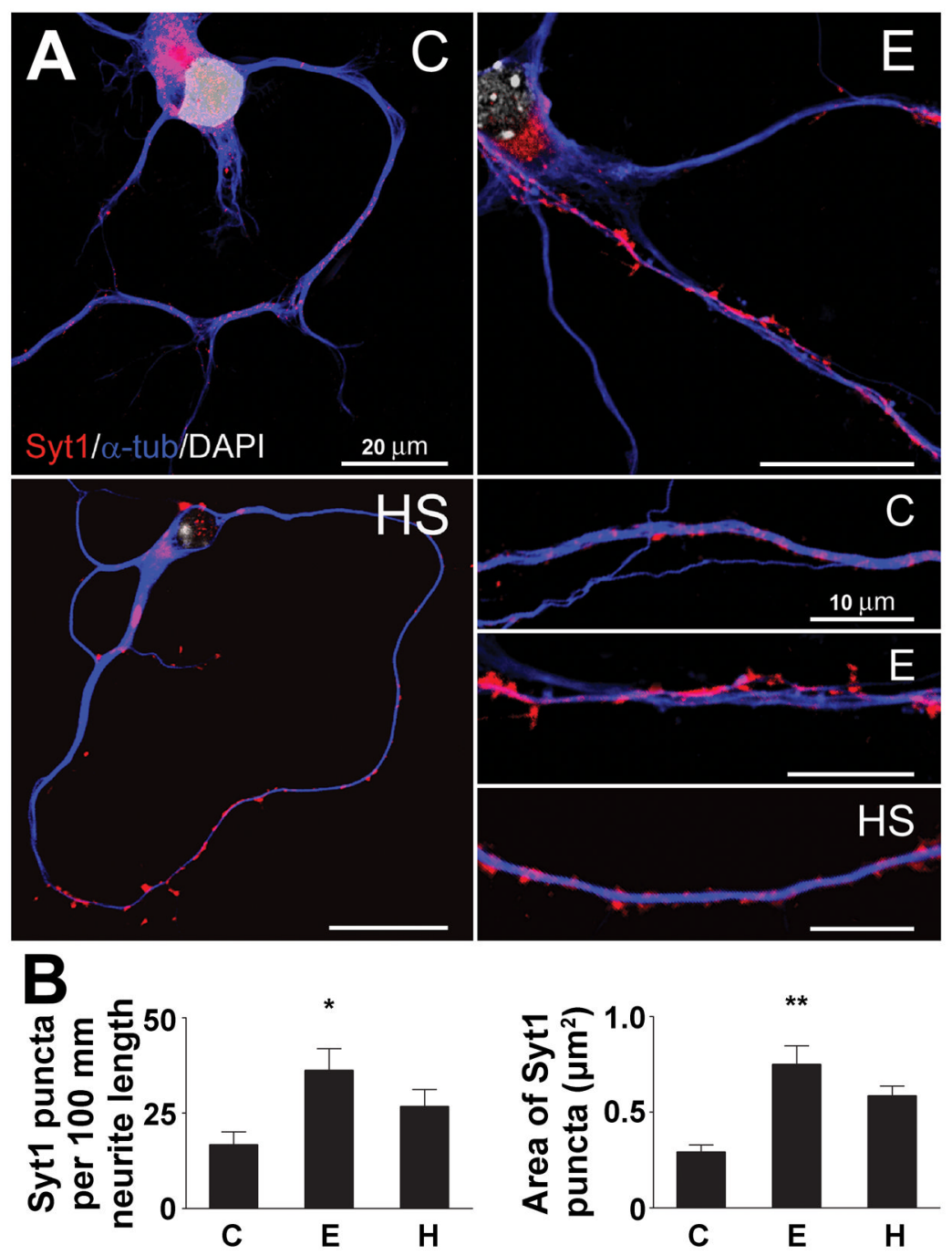

Figure 3. Ethanol treatment increases Syt1-positive clusters of immunoreactivity in cortical neurons

$\boldsymbol{A}$, Visualization of cortical neurons after treatment with $60 \mathrm{mM}$ ethanol (E), a $42^{\circ} \mathrm{C}$ heat shock (HS) or vehicle control (C). Immunocytochemistry was performed using anti-Syt1 and anti-a-tubulin antibodies, and DAPI nuclear staining

$\boldsymbol{B}$, Increase in the number and size of Syt1-positive clusters after neurons were treated with ethanol. The graphs show the number of Syt1-positive clusters per $100 \mathrm{~mm}$ neurite length and the average cluster size after neurons were exposed to $60 \mathrm{mM}$ ethanol (E), a $42^{\circ} \mathrm{C}$ heat shock treatment (HS) or vehicle control (C). The quantification was performed with ImageJ software. The data were compared with control values by 1-way ANOVA with Dunnett's multiple-comparison post-hoc test ( $\mathrm{n} \geq 6$; length: $\mathrm{F}=3.750, \mathrm{df}=22$; area: $\mathrm{F}=4.977$, $\mathrm{df}=$ 569). All data are mean \pm SEM (* significantly different at the level of $p<0.05, * * p<$ $0.01)$. 

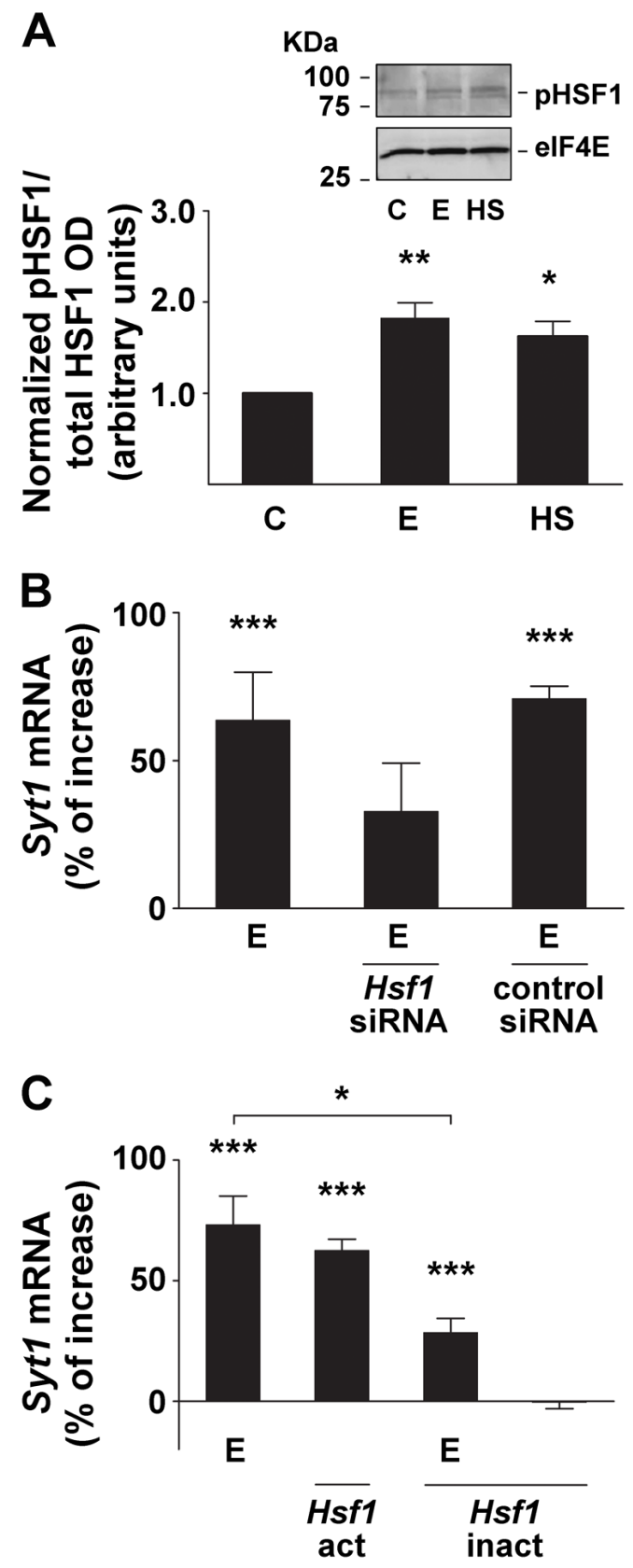

Figure 4. The induction of the Syt1 gene by ethanol requires transcriptionally activated heat shock factor 1 protein

$\boldsymbol{A}$, Increase in phosphorylated HSF1 (pHSF1) protein after ethanol and heat exposure. The inset shows a representative Western blot of the relative abundance of pHSF1 protein in neurons treated with $60 \mathrm{mM}$ ethanol (E) or $42^{\circ} \mathrm{C}$ heat shock (HS) for 2 hours of exposure, or vehicle control (C). The bar graph represents normalized optical density (OD) of pHSF1 relative to total HSF1 OD. Ethanol and heat shock data were compared with control by 1way ANOVA, with Dunnett's multiple-comparison post-hoc test $(\mathrm{n} \geq 3 ; \mathrm{F}=10.15, \mathrm{df}=8)$. All data are mean \pm SEM (* significantly different at the level of $p<0.05$, ** $p<0.01$ ). $\boldsymbol{B}$, Knock-down of heat shock factor 1 (HSF1) inhibits Sytl gene induction by ethanol. Pretreatment of neurons with $H s f l$ siRNA reduced the effects of $60 \mathrm{mM}$ ethanol exposure 
(E) on Syt1 gene transcription, while pretreatment with control siRNA had no effect on Syt1 gene induction by ethanol. The data were normalized to Actb cDNA, and the ethanolexposed samples were compared with control samples using 1-way ANOVA and Dunnett's multiple-comparison post-hoc test $(\mathrm{n} \geq 6 ; \mathrm{F}=17.50, \mathrm{df}=23)$. All data are mean $\pm \mathrm{SEM}$ (*** significantly different at the level of $p<0.001$ ).

$\boldsymbol{C}$, Stimulation of $S y t 1$ gene expression by ethanol is mediated by activated heat shock factor 1 (HSF1). Cortical neurons transfected with a constituitively active $H s f l$ construct (Hsflact) showed an increase in Syt1 mRNA expression, similar to the gene's induction by 60 $\mathrm{mM}$ ethanol (E). Transfection of a constituitively inactivated form of Hsfl (Hsfl-inact) reduced the effects of ethanol on Sytl gene induction. Hsfl-inact transfection alone had no effect on Syt 1 expression. The data were normalized to Actb cDNA, and the treated samples were compared with control samples using 1-way ANOVA and Dunnett's multiplecomparison post-hoc test $(\mathrm{n} \geq 6 ; \mathrm{F}=36.56, \mathrm{df}=159)$. All data are mean $\pm \mathrm{SEM}(*$ significantly different at the level of $p<0.05, * * * p<0.001$ ). 


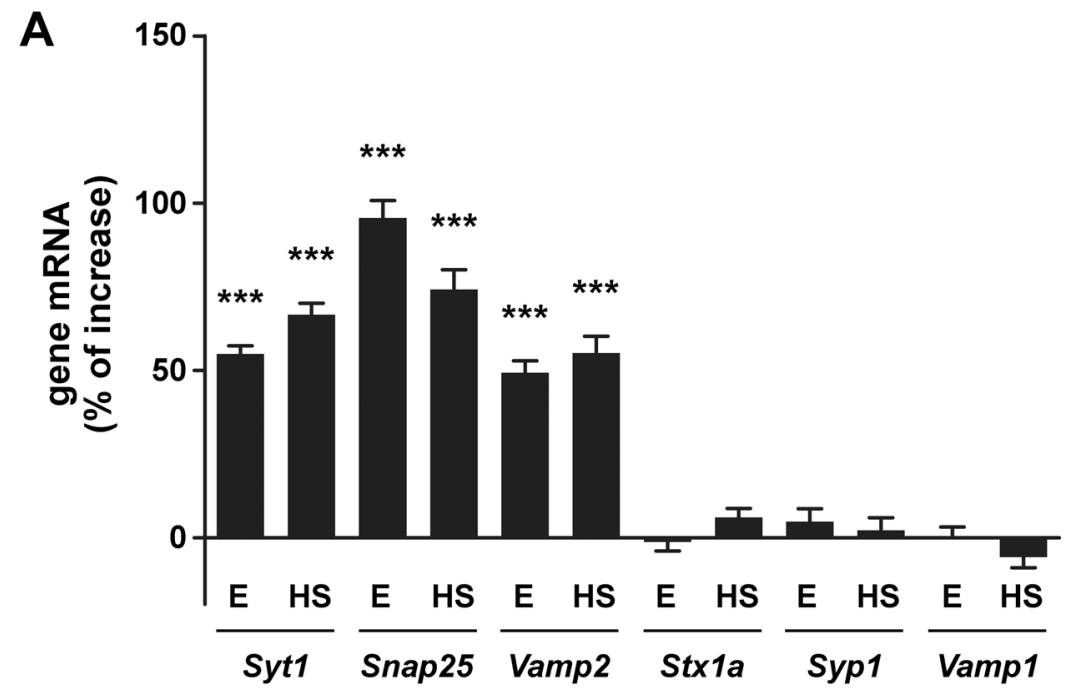

B
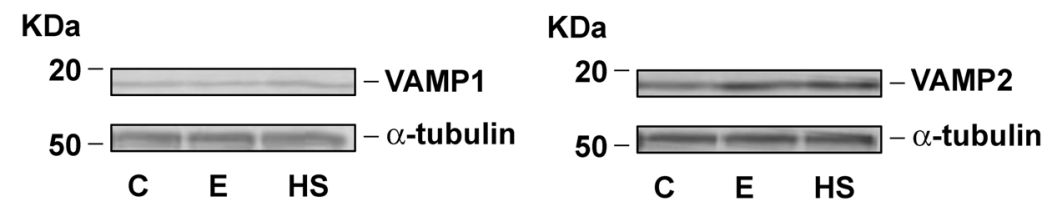

C Syt1 (514 kb)

E1 E2-5 E6 E7-8
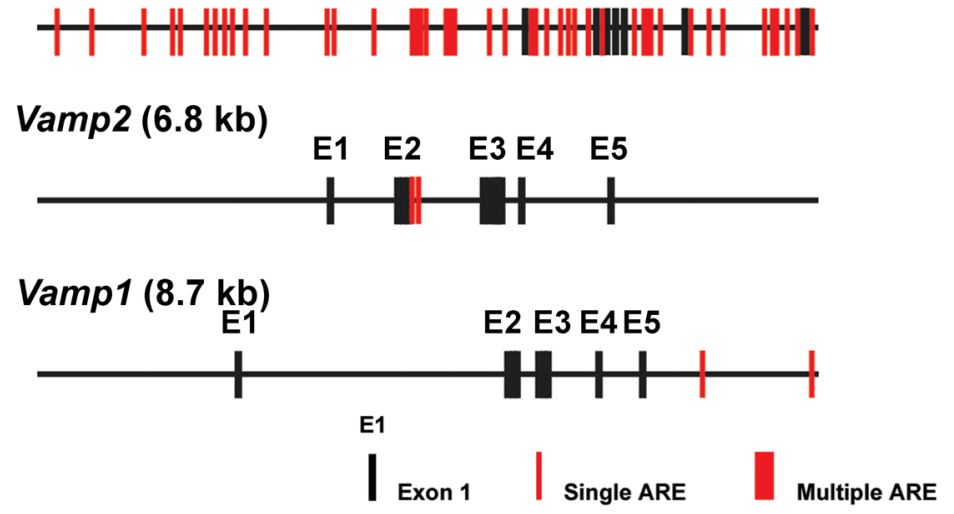

Figure 5. Ethanol induction of synaptic vesicle fusion machinery gene and protein expression levels correlate with ARE position

$\boldsymbol{A}$, Ethanol and heat shock induce a subset of genes encoding synaptic vesicle fusion proteins. Snap 25 and Vamp2 mRNA expression levels increased after treatment for 1 hour with $60 \mathrm{mM}$ ethanol (E) or $42^{\circ} \mathrm{C}$ heat shock (HS), as measured by qPCR. Stxla, Sypl, and Vampl mRNA levels were not significantly altered by either treatment. The data were normalized to Actb cDNA, and the treated samples were compared with control samples using 1-way ANOVA and Dunnett's multiple-comparison post-hoc test, $\mathrm{n} \geq 6$. All data are mean \pm SEM (*** significantly different at the level of $p<0.001)$.

$\boldsymbol{B}$, Ethanol and heat shock increase VAMP2 protein levels, but have no effect on VAMP1expression. The graphs shows the relative abundance of VAMP1 and VAMP2 
protein in neurons treated with $60 \mathrm{mM}$ ethanol (E) or $42^{\circ} \mathrm{C}$ heat shock (HS) for 2 hours of exposure, or vehicle control (C).

$\boldsymbol{C}$, Schematic representation of the Syt1, Vamp2 and Vamp1 genes reveals that the two ethanol sensitive genes contain alcohol response elements (ARE) in their upstream promoter regions. These putative regulation sites may bind heat shock factor 1 (HSF1) to mediate gene induction by ethanol. In Vampl, an ethanol-insensitive gene, the ARE sequences are all located far downstream in the 3'-untranslated region of the gene, while the ARE in Vamp2 are located in the second intron. The relative positions of the introns, exons and ARE are conserved in this illustration. 